\title{
Representing Hotspots of Evolutionary History in Systematic Conservation Planning for European Mammals
}

\author{
Anni Arponen and Laure Zupan
}

\begin{abstract}
Systematic conservation planning deals with cost-effective allocation of conservation funds. There are diverse ways in which evolutionary history could be included in prioritization, but here we considered it at the local scale, valuing higher the locations where the local community has high phylogenetic diversity, while still aiming at maximizing overall species representation. We conducted the prioritization with the Zonation software for spatial conservation planning.

We prioritized areas for conservation in Europe using distribution data and phylogenies for 275 mammal species. We prioritized areas in Europe for conserving hotspots of evolutionary history. For comparison we made analyses with species occurrences alone. Analyses were done for the whole region and for each country separately. We explored the impacts of tree uncertainty, and analyzed how well existing protected areas performed with respect to Zonation priorities.

Our findings indicate that some hotspots of evolutionary history are missed by species-based prioritization, unless specifically accounted for. Uncertainty in spatial priorities caused by variation in phylogenetic tree structure was a minor concern for prioritization. Protected areas did not perform well when assessed against the Zonation priorities for species or for phylogenetic diversity, although highest national scale priorities had almost twice as much area protected as the overall average.
\end{abstract}

We emphasize that the chosen goals and analysis setups have strong impacts on spatial priorities and therefore care must be taken in defining them appropriately. But regardless of setups, the gap between the current conservation efforts and spatial prioritization outcomes is typically greater than the difference between including and excluding phylogenetic diversity. Therefore the focus should be on increasing the role of spatial analyses in practical conservation, but whenever

\footnotetext{
A. Arponen $(\square)$

Department of Biosciences, Faculty of Biological and Environmental Sciences, University of Helsinki, P.O. Box 65 (Viikinkaari 1), FI-00014 Helsinki, Finland

e-mail: anni.arponen@ helsinki.fi
}

L. Zupan

Laboratoire d'Ecologie Alpine, UMR-CNRS 5553, Université J. Fourier,

Grenoble I, 53, 38041 Grenoble cedex 9, France

e-mail: laure.zupan@gmail.com

R. Pellens, P. Grandcolas (eds.), Biodiversity Conservation and Phylogenetic

Systematics, Topics in Biodiversity and Conservation 14,

DOI 10.1007/978-3-319-22461-9_13 
feasible, also including evolutionary history in the analyses, because evolutionary history is not always well represented by targeting species for conservation.

Keywords Phylogenetic diversity - Quadratic entropy • Spatial prioritization • Zonation

\section{Introduction}

Systematic Conservation Planning Protected areas around the world have typically been established in areas of low competing interests, which is not ideal from the perspective of biodiversity conservation (Pressey et al. 1993). Such biased allocation may even lead to existing protected areas performing worse than randomly chosen areas in representing diversity (Ferrier 2002). The realization that conservation would benefit from cost-effective practices led to the development of the field of Systematic conservation planning (Box 1, Margules and Pressey 2000; Margules and Sarkar 2007). More than 20 years of development have led to the integration of numerous aspects to the approach adding to its realism. In particular, the spatial prioritization for assessing the existing conservation areas and selecting new ones have become comprehensive and efficient, and nowadays they also provide more user-friendly graphical user interfaces, which has facilitated their broad use for practical conservation planning purposes (Ball et al. 2009; Moilanen et al. 2009).

Evolutionary History in Conservation Phylogenetic diversity or species originality are often mentioned as important for conservation (Rosauer and Mooers 2013; Winter et al. 2013), and the history of such discussion goes back already a few decades (Vane-Wright et al. 1991; Faith 1992). Evolutionary history is often quantified in community ecology for the purpose of understanding the diversity of current species distributions (Davies and Buckley 2011; Fritz and Rahbek 2012) or the potential functioning of ecosystems (Cadotte et al. 2012), whereas applications to conservation have remained limited.

\section{Box 1}

The process of systematic conservation planning as described by Margules and Pressey (2000).

1. Compile data on the biodiversity of the planning region

2. Identify conservation goals for the planning region

3. Review existing conservation areas

4. Select additional conservation areas

5. Implement conservation actions

6. Maintain the required values of conservation areas 
Numerous indices have been developed to measure the originality of species (Vane-Wright et al. 1991; Pavoine et al. 2005a; Isaac et al. 2007), or phylogenetic diversity (Faith 1992; Schweiger et al. 2008; Pavoine and Bonsall 2011; Faith chapter "The PD Phylogenetic Diversity Framework: Linking Evolutionary History to Feature Diversity for BiodiversityConservation"). The former measures assign a value for each species based on their dissimilarity from other species, whereas the latter look at an assemblage of species as a whole.

Both types can be used in spatial conservation prioritization (Arponen 2012). Originality can be used for weighting species differently, whereas diversity indices can be used at different scales: either for measuring the diversity of all species across a network of protected areas, or for preferentially selecting areas with high local, alpha-level diversity of the community. Their use has been rare in published studies of spatial conservation prioritization. Arponen et al. (2005) used species weights based on species originality in conservation prioritization for plants in Finnish herb-rich forests. There are also some examples of considering assemblagelevel phylogenetic diversity across a network of sites: The "Phylogenetic Diversity" of Faith (1992) has been used for conservation prioritization with birds (Rodrigues and Gaston 2002) and plants (Forest et al. 2007) in South Africa, as well as in a global analysis for mammals (Rodrigues et al. 2011). Instead of spatial prioritization of areas for protection, evolutionary history has been considered much more commonly in other kinds of conservation contexts (reviewed in Arponen 2012), such as creating priority lists of species for conservation. For example, Isaac et al. (2007) introduced the "Evolutionary distinctiveness" measure for species and used it in combination with extinction risk data to assign priorities for species in the EDGE program (see also May-Collado et al. chapter "Global Spatial Analyses of Phylogenetic Conservation Priorities for Aquatic Mammals"; Schnell and Safi chapter "Metapopulation Capacity Meets Evolutionary Distinctness: Spatial Fragmentation Complements Phylogenetic Rarity in Prioritization").

To our knowledge, phylogenetic diversity has not been used at the scale of local communities in spatial conservation prioritization. The use of alpha-level phylogenetic diversity is based on the assumption that it would correlate with ecological processes better than species richness of the community (Forest et al. 2007), and therefore work as an indicator for functional diversity when species traits data are missing. This is based on the idea that phylogenetically distinct species are likely to be functionally different (Cadotte et al. 2008), although this assumption has also been challenged (Mouquet et al. 2012). For this purpose, phylogenetic diversity indices that account for species abundances (Chao et al. 2010; Chao et al. chapter "Phylogenetic Diversity Measures and Their Decomposition: A Framework Based on Hill Numbers") might be more suitable than the ones that consider only presences and absences of species (such as Faith 1992): from the perspective of ecosystem function, viable populations and sparse individuals of a species should not be considered equally important.

Case Study on European Mammals Mammals are a fairly well known group of species regarding their ecology, distributions as well as phylogeny. Nevertheless, their phylogenies are not fully resolved, but contain polytomies. Resolving the 
polytomies randomly results in variation among different trees, but having good spatial distribution data provides a good opportunity for investigating the influence of such uncertainty on spatial conservation prioritization. Mammals are also considered to be of high conservation interest due to their public appeal (Smith et al. 2012). They were the first focal taxon of the EDGE programme (Isaac et al. 2007), which was a pioneering endeavor to bring highly threatened and evolutionarily unique species to the limelight and to improve their conservation.

We conducted spatial prioritizations for European mammal conservation with the Zonation conservation planning software. We compared traditional, species based prioritization to one where alpha-level phylogenetic diversity, measured as the equivalent number of Rao's quadratic entropy, was allowed to influence site value through using its inverse as cost in the analyses. Because a continental scale analysis may not be politically feasible, we repeated both analyses at national scales, where Zonation performs identical prioritization but for each country separately. For mammals there is still some uncertainty related to the structure of the phylogeny. We acquired 100 different trees and ran Zonation analyses for each of them, comparing the similarities of outcomes to each other. We analyzed the trade-offs between species representation and the equivalent number of Rao's quadratic entropy in the solutions. Finally, we analyzed the performance of the current protected area network in representing hotspots of evolutionary history for mammals, as well as in representing species, both at the European and at national scales.

\section{Material and Methods}

European Mammal Distributions We used data on the spatial distribution of european terrestrial mammals described in Maiorano et al. (2013). The primary data were extents of occurrence (EOOs) of the species occurring in Europe and Turkey obtained from the Global Mammal Assessment (http://www.iucnredlist.org/initiatives/mammals; accessed 15 August 2013 (IUCN 2012)). To refine EOOs and remove potential false presences, habitat requirements were used in an expert-based modelling approach. More specifically, for each species, habitat requirement was defined by experts (G. Amori, D. Russo and L. Boitani) and published literature (see Maiorano et al. 2013 for the full list of references) based on three environmental variables: land cover, elevation and distance to water. For each species, data collected were used to assign a suitability score $(0$, unsuitable; 1 , secondary habitat and 2 , primary habitat) to each of the 46 GlobCover land-use/land-cover classes. Elevation and distance to water were then combined to the habitat suitability score to refine the available EOOs and obtain current distribution with a cell size of $300 \mathrm{~m}$ resolution. The models were validated with help of field data (see Maiorano et al. 2013 for more details). From these 288 species we used 275 for which phylogenies were available. 
As running the phylogenetic analyses and Zonation prioritization at $300 \mathrm{~m}$ resolution would have been too demanding for the equipment available at the time, we scaled up the species distributions following a regular grid of $10^{\prime}$. As a value for each $10^{\prime}$ cell, we kept the percentage of $300 \mathrm{~m}$ cells considered as either 1 (primary habitat) or 2 (secondary habitat), and we refer to this value as "the proportion of suitable area" hereafter. For aesthetic reasons, all the maps presented hereafter have been projected using the Lambert conformal conic projection (UTM zone 34).

Mammal Phylogenies Phylogenetic data for mammals were based on the supertree of Bininda-Emonds et al. (2007) updated by Fritz et al. (2009). We used 100 fully resolved phylogenetic trees, where polytomies were randomly resolved applying a birth-death model to simulate branch lengths (Kuhn et al. 2011).

Protected Areas We used the WDPA dataset on protected areas (UNEP 2010) categories I-IV (I: Strict nature reserve or wilderness area, II: National park, III: Natural monument or feature and IV: Habitat/Species management area) excluding the categories that are generally considered less beneficial for biodiversity conservation (categories V and VI), and areas where the category was either 'not reported' or 'not applicable'. We used the proportions of area protected in each cell for our analyses of overlap of Zonation priorities with protected areas. WDPA data are polygons. As Zonation operates with raster data, we transformed the polygons into a raster, following the same grid as the species distribution data $\left(10^{\prime}\right.$ cells regular grid). To do so, we overlapped the polygons on the grid and retained the proportion of area protected in each grid cell.

Measuring Phylogenetic Diversity To measure the phylogenetic diversity at each cell, we used the Rao's quadratic entropy (Rao 1982), an index of alpha-diversity, which is extended to account for the pair-wise dissimilarities of species:

$$
Q E=\sum_{i=1}^{S} \sum_{j=1}^{S} d_{i j} p_{i c} p_{j c}
$$

$\mathrm{d}_{i j}$ is derived from the ultrametric phylogenetic tree (Pavoine et al. 2005b) and corresponds to the phylogenetic dissimilarity between each pair of species $i$ and $j . p_{i}$ and $p_{j}$ are the respective proportions of suitable habitat for the species $i$ and $j$ available in the $10^{\prime}$ pixel $c$. It is now recognized in the literature that the values of most of diversity measures (like the Rao's quadratic entropy) do not behave intuitively because they do not satisfy the "replication principle" (Jost 2007; de Bello et al. 2010; Chao et al. 2010; Leinster and Cobbold 2012; Chao et al. chapter "Phylogenetic Diversity Measures and Their Decomposition: A Framework Based on Hill Numbers"). The replication principle (or "doubling property") states that if we pool two equally diverse and equally large groups with no shared species, the total diversity should be two times the diversity of a single group (Chao et al. 2010; Chao et al. see their Fig. 2 in chapter "Phylogenetic Diversity Measures and Their Decomposition: A Framework Based on Hill Numbers"). To make the Rao's 
quadratic entropy behave this way, we need to transform it into an equivalent number through a simple algebra step (1/(1-QE), Jost 2007). The outcome is a raster layer with the value of $\mathrm{QE}$ in equivalent number for each of the $10^{\prime}$ pixels with the same spatial extent and resolution as the mammal distribution data.

The Zonation Approach Zonation is a spatial prioritization software meant to be used as a decision support tool (Moilanen et al. 2009). While other approaches typically select a fraction of the landscape according to a pre-determined target, e.g. $10 \%$ of species distributions, or maximize what is achieved with a pre-determined budget, Zonation instead ranks all cells in the entire landscape in the order of conservation value. A Zonation solution can be used to identify any best (or worst) fraction of the landscape.

The ranking is based on the evaluation of range size normalized richness of biodiversity features in each cell (Moilanen et al. 2005, 2011). In plain words, this means that features (e.g. species) with broad distributions contribute very little to the conservation value of a single cell, whereas narrowly distributed species substantially increase the conservation value of the cells they occupy. At every iteration (removal of one cell) Zonation recalculates the conservation value for the remaining cells based on the remaining feature distributions, which become smaller with each iteration. Thus, Zonation removes first cells with few, broadly distributed features, and during the ranking these features become rarer and rarer in the remaining landscape. As an outcome, the remaining highest priority fraction of the landscape will contain the cells with high species richness and narrow endemics.

Zonation provides two options as cell-removal rules that determine how the marginal value of a cell is calculated (Moilanen et al. 2005; Moilanen 2007). The additive benefit function approach allows for more flexible trade-offs to occur between features, because it considers cell value as the sum over benefit functions of representation of the features in the cell. This means that narrowly distributed species in species poor (or expensive) cells may be traded off against species rich cells. We chose to use the Core-area cell removal rule, which defines the cell value based on the most valuable occurrence over all species in the cell. This means that if a cell contains a large fraction of the range even for only one species, it will get high value, regardless of the species richness in the cell. This way the core areas of all species' ranges are retained in the highest priority fraction of the landscape. As species distribution data, we used the raster layers of proportion of suitable habitat per cell for each species, as described above in the section "European mammal distributions".

Even though Zonation does not consider phylogenetic data by default, it offers also options for accounting for evolutionary history in the prioritization. For example, species could be weighted based on their evolutionary distinctiveness either globally, or with different region-specific weights (Moilanen and Arponen 2011). Alternatively, locations can be weighted based on the phylogenetic diversity of the local community. In this case study we focus on the latter approach. Technically this happens through defining a "cost layer" as inversely proportional to the diversity. This way a cell with one-fifth of the phylogenetic diversity of another cell is 
considered five times as costly to protect, lowering its position in the Zonation ranking. The cost layer can be scaled differently according to how much importance is given to phylogenetic diversity. The equivalent numbers of Rao's quadratic entropy values went from ca. 1 to 7, and the direct inverse was used in our "medium weighting" (that is, cost goes from 0.14 to 1), and this scale was halved ("low weights", $0.28-1$ ) and doubled ("high weights", 0.07-1) to test for sensitivity to this parameter (see Fig. 1 for analysis setups).

The latitudinal gradients in species richness and range sizes cause the spatial priorities in analyses at any scale to be concentrated in the more species rich lower latitude areas (Eklund et al. 2011; Moilanen et al. 2013). Even though cost-effective from the perspective of species conservation, focusing conservation efforts into these regions only would be very difficult for many reasons (see section "Discussion and Conclusions"). Therefore we also performed an analysis where countries were considered as independent administrative units, each aiming to conserve the diversity within their borders. This is implemented through the Administrative units analysis in Zonation (Moilanen and Arponen 2011). The analysis would allow for a compromise solution between purely European-scale and purely national-scale analyses, but for our analytical purposes, we chose the extreme cases only. A national-scale prioritization provides an interesting reference for comparison to protected areas. We did this for one tree only. Thus, we ended up with four main Zonation solutions to assess protected area performance regarding the representation of species and phylogenetic diversity at both European and national scales (Fig. 1).

\section{Case Study Setup}

\section{Results}

Spatial priorities in the European analyses were strongly concentrated around the southern parts as well as eastern border of the study region (Fig. 2a, b). Spatial priorities between the basic Core-area prioritization and the variants where phylogenetic diversity as the equivalent number of Rao's QE was included are extremely similar in some regions, but contain some rather dramatic differences in specific, especially northern parts of Europe (Fig. 2a, b). Spearman rank correlations between the rank values in the basic Core-area solution and the three weighting variants of the equivalent number of Rao's QE were 0.93, 0.91 and 0.89 , for the low, medium and high weight scales, respectively.

We repeated the basic and phylogenetic diversity weighted analyses at the national scale, where Zonation performed the prioritization separately for each country (Fig. 2c, d). Here the priorities were forced to be evenly distributed among the countries, such that e.g. the best $10 \%$ of the landscape consisted of the best $10 \%$ in each country. Such priorities are much more scattered across Europe, and concentrated around country borders. 


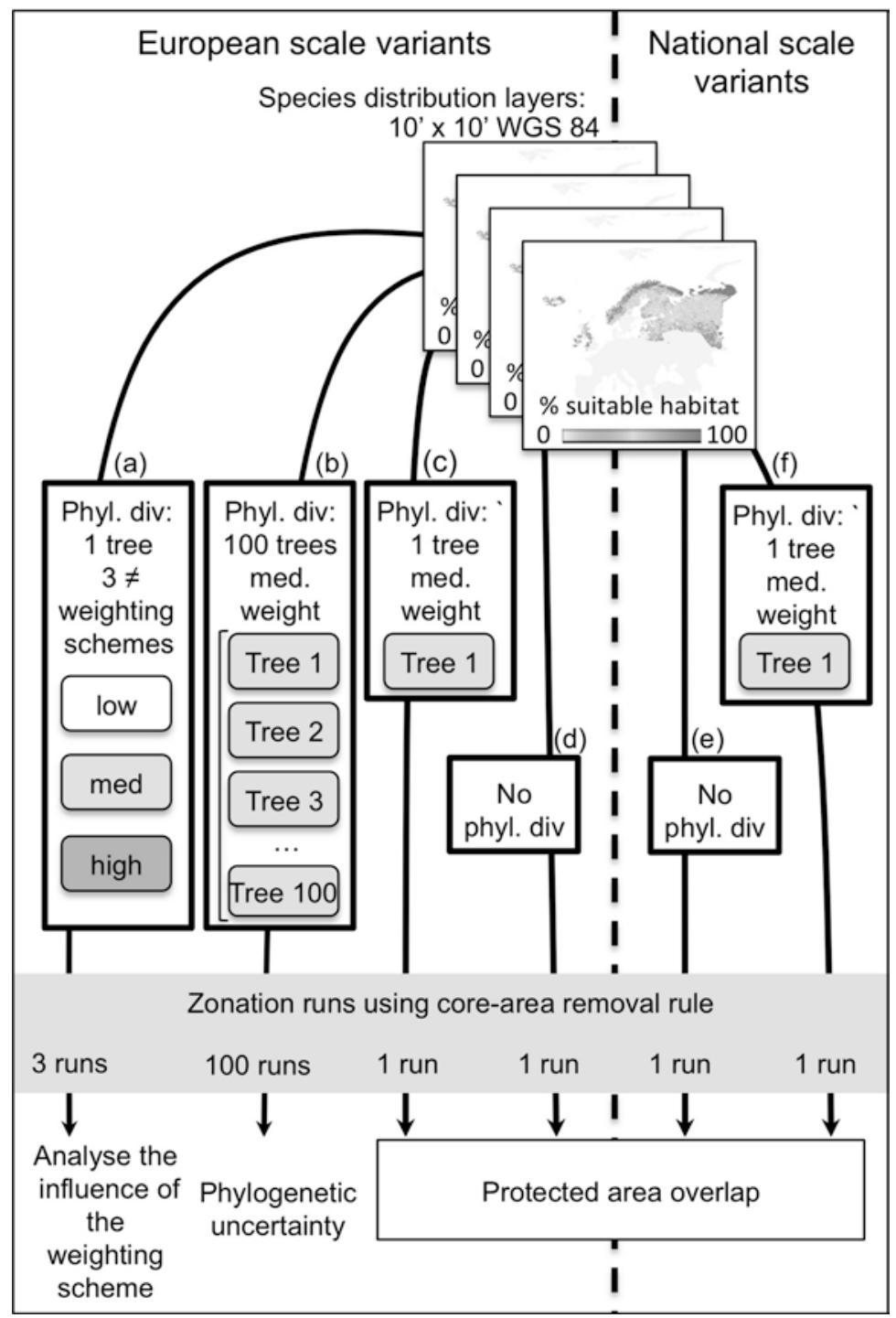

Fig. 1 Diagram representing the flow of our analyses. The left part of the figure illustrates the analyses we ran at the European scale while at the right of the dashed line are the analyses conducted at the national level. In all analyses, the data input for the species were the proportion of suitable habitat (1 raster layer per species). We first (a) tested three different weightings for the phylogenetic diversity measured as the equivalent number of Rao's quadratic entropy (low, medium and high weighting, see main text) to assess whether this was influencing the prioritization results. In a second step (b) we ran Zonation 100 times using cost layers corresponding to the 100 different phylogenetic trees. We followed this procedure to evaluate the influence of the tree structure variation on the prioritization results. The remaining analyses were dedicated for the evaluation of the current protected areas network. We used only one cost-layer (corresponding to the equivalent number of Rao's quadratic entropy extracted from tree 1 and a medium weighting) to evaluate the protected area network at the (c) European scale and (f) the national scale. Finally we ran Zonation without any phylogenetic diversity data to assess the representation of species within the protected areas network at (d) European scale and (f) national scale. Abbreviations: med medium, phyl. div phylogenetic diversity measured as the equivalent number of Rao's quadratic entropy 

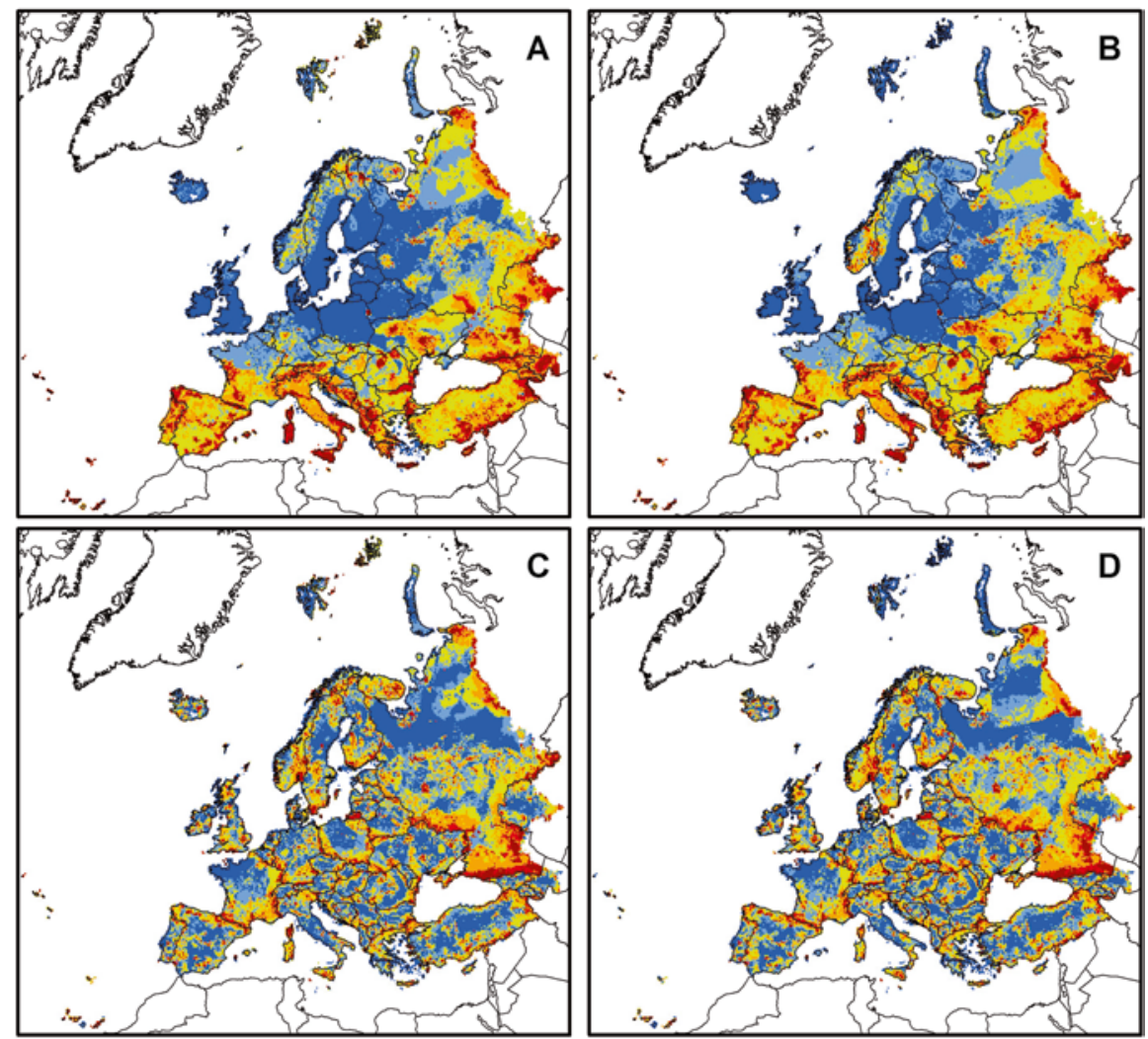

\section{Best fraction of landscape}

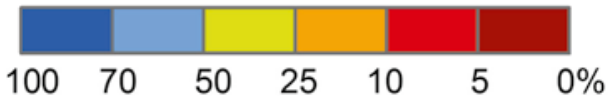

Fig. 2 Zonation priority maps for mammals in Europe. The red tones represent the best $10 \%$ of the solution and blue tones indicate the lowest $50 \%$ of cells. (a) Is the basic, core-area Zonation solution for our data where conservation value in Zonation optimization is only based on species richness normalised by range size. (b) Is the Zonation solution where the conservation value of a cell is weighted with the medium variant of the phylogenetic diversity, i.e. the inverse of the equivalent number of Rao's quadratic entropy for the local community in each cell is used as cell costs. (c) Shows the national level basic Zonation priorities and (d) is the national analysis with phylogenetic diversity included

The three phylogenetic weightings give very similar results. The pair-wise Spearman rank correlations between these differently weighted Zonation analyses were very high (low-medium: 0.9965, low-high: 0.9916, and medium-high: 0.9987). Therefore, in the following analyses we used the medium weighting only, which corresponds to using the inverse of the equivalent number of Rao's QE directly as cell cost (see Fig. 1). 


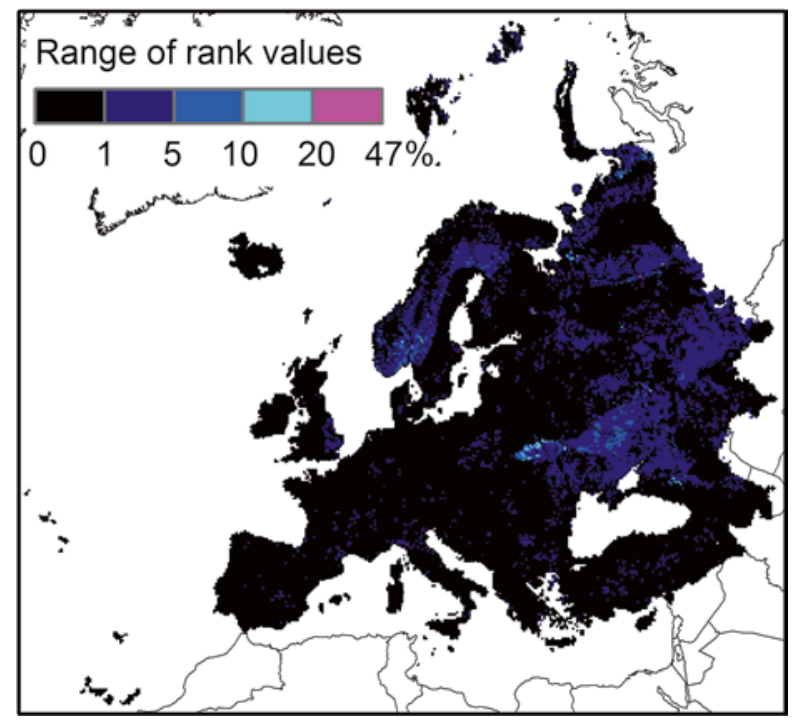

Fig. 3 The range of variation in rank values among the 100 Zonation solutions done with different phylogenetic trees. Large majority of areas have very consistent rank values, with variation lower than $1 \%$ (i.e. always placed within the same $1 \%$ fraction in the Zonation ranking; black in the map). In some regions the variation is broader, but still keeping within the same $10 \%$ fraction in Zonation (medium blue). Only very small regions have variation from 10 to $20 \%$ (light blue), and only some sparse cells go through more dramatic changes in priority when different tree structures are considered, with variation up to $47 \%$ (pink cells)

Similarly, pair-wise Spearman rank correlations for Zonation solutions done with the different 100 phylogenetic trees were also very high. The mean pair-wise correlation was 0.99985 and even the lowest pair-wise correlation was 0.99934 . There were only a few regions across the study area where the rankings were not consistent (Fig. 3).

We also tested whether the uncertainty of tree structure was related to the position in Zonation rank, that is, whether there may have been more or less uncertainty associated with top ranking cells. Pair-wise Spearman rank correlations of uncertainty with each of the main Zonation variants gave weak, positive correlations of 0.10 for the basic solution, 0.12 for the phylogenetic diversity analysis, 0.08 for the basic national scale analysis, and 0.07 for the national scale phylogenetic diversity analysis. As the tree uncertainty seemed to play a very minor role in the prioritization outcome, in the following analyses we used one tree only (see Fig. 1).

To assess how well the Zonation priorities covered the different species' ranges, we plotted the proportions of species ranges retained in the landscape at different fractions of cell removal (Fig. 4). The median of representation is higher for the analysis with phylogenetic diversity than for the one without (Fig. 4, black squares). This may seem surprising, but is explained by the fact that Rao's QE correlates with species richness. Looking into the corresponding values for individual species 

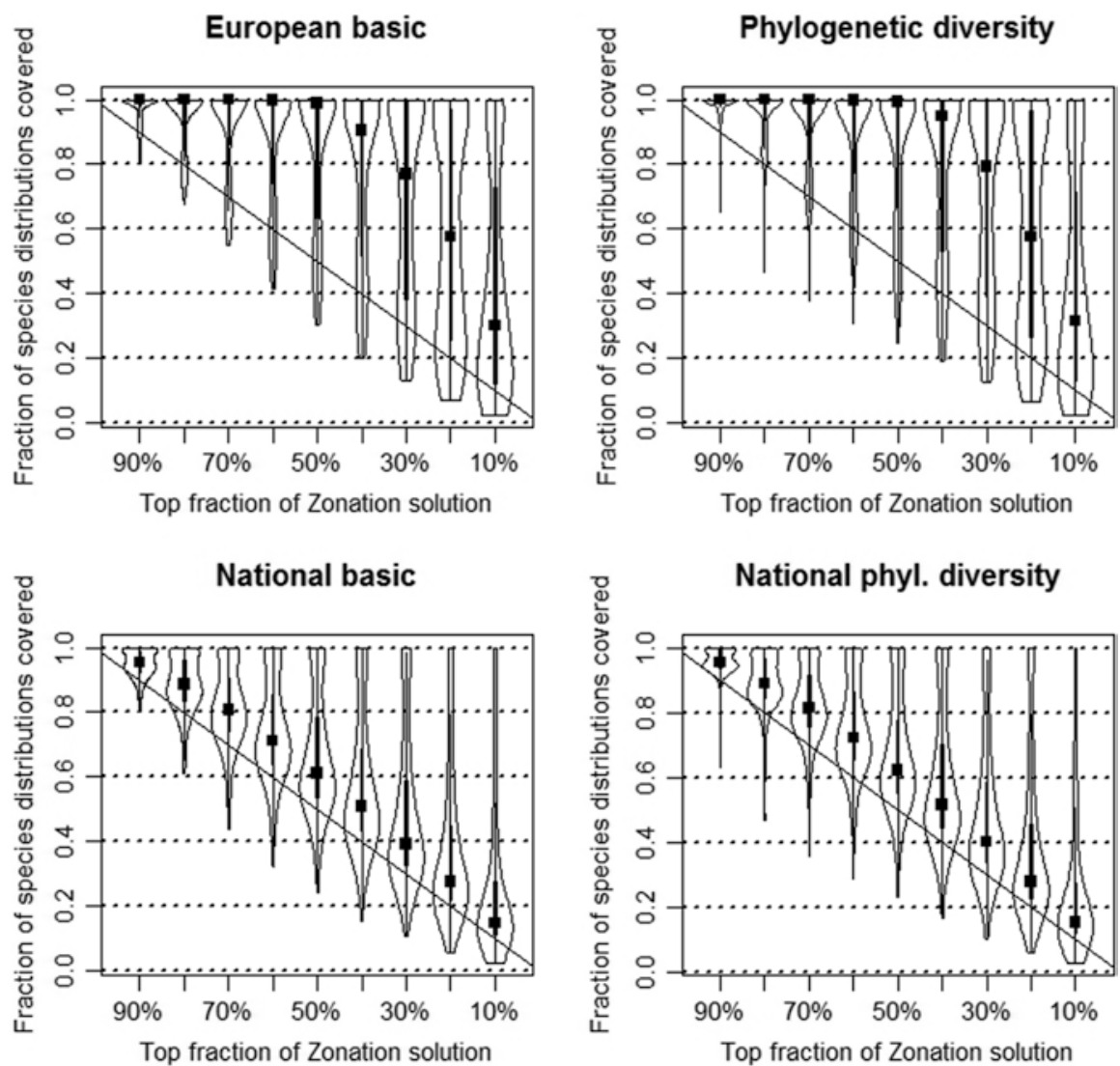

Fig. 4 Proportions of species distributions retained (y-axis) in different top fractions of the landscape (x-axis). The black squares represent the median value across all species, which are surrounded by vertically plotted density distributions of all species' values around the median. For example, in the basic Zonation solution, the top $20 \%$ of the landscape covers more than $55 \%$ of the ranges for half of the species, but there are also (broadly distributed) species with only ca. $10 \%$ of their ranges covered. A random selection at the continental scale would result in a 1:1 diagonal line for the medians (solid line)

(illustrated by the density distributions drawn around the medians in Fig. 4) reveals a very subtle trade-off: The basic core-area Zonation retains species representations more evenly, as it should by definition, whereas the phylogenetic diversity solution loses larger fractions of some species' ranges earlier on in the cell removal process (longer downward tails in the density distributions at lowest $50 \%$ fractions). In other words, with the phylogenetic diversity weighting the protection of some species is traded off against protection of locations with higher phylogenetic diversity. But as this tradeoff is minor and most visible at poorest fractions of the landscape, it is unlikely to be of concern for practical conservation. 


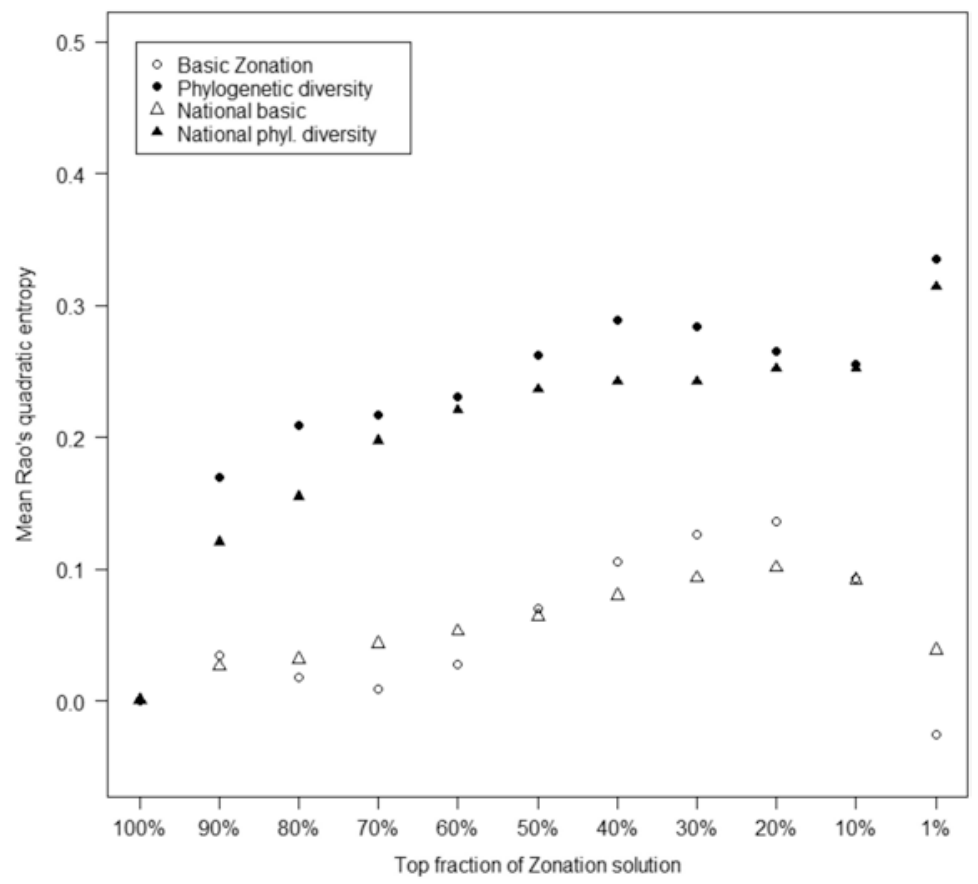

Fig. 5 Mean phylogenetic diversity measured as the equivalent number of Rao's QE across cells at different top fractions of the landscape according to Zonation. The values have been standardized from an original mean QE of 5.06 across all cells. The different prioritizations converge at top fraction equal to 1 , as that represents the mean value across all cells in the landscape. If cells were removed in random order, the points would form a flat line at this level

A major difference can be seen between the analyses at different spatial scales. When going from European to national priorities, the median representation values drop substantially, by even ca. $40 \%$ (Fig. 4). Same pattern arises at the national scale from inclusion of phylogenetic diversity: again some species lose more of their ranges for the benefit of others that occur in locations with higher values of the equivalent number of Rao's QE.

To look at evolutionary history maintained by the Zonation solutions, we plotted the mean equivalent number of Rao's QE for the cells at different top fractions of the rankings (Fig. 5). We observed as an overall general trend that the mean QE is increasing as cells are removed from the landscape (from 100 to $1 \%$ in the X-axis) for any selection procedure (with or without including phylogenetic diversity as selection criteria). This is caused by the positive correlation between QE and species richness. By default, Zonation values high species richness cells which will tend to be prioritized, and those cells are also more likely to have high QE values than species poor cells. The very highest priorities (top $1 \%$ in Fig. 5) again diverge from this trend for the solutions that do not consider QE explicitly, because here Zonation tries to maintain a representation for as many species as possible, and thus the complementarity of species compositions overrides the importance of richness, and correlation with the QE weighted solutions disappear. 
The mean equivalent number of Rao's QE retained by each fraction of the landscape is higher when phylogenetic diversity is accounted for (black-filled symbols are higher than empty symbols). This means that including phylogenetic diversity as a prioritization criterion improves the outcome of the Zonation solution from the perspective of evolutionary history. Our results also highlight that the scale at which the prioritization is conducted (European vs. National) does not appear to have a consistent impact on the mean QE retained in each fraction of the landscape (same colored symbols are close to each other for a given fraction). In other words, the choice whether to conduct a prioritization at the country level or at the continent level does not influence how much phylogenetic diversity is retained.

We overlaid the Zonation rankings with maps of existing protected areas to see how well the priorities and protected areas coincide. The protected areas we considered in our analyses (WDPA categories I-IV) cover a total of $7.8 \%$ of the land area in the study region. We compared them to the same amount of land area prioritized by the Zonation variants (Fig. 6), i.e., $7.8 \%$ top fraction of the Zonation solutions. A large majority of currently protected land is not considered of high priority by any of the Zonation variants (light blue areas), and conversely, much of the Zonation priorities are unprotected (yellow-orange tones). The best matching areas are shown in red, and are sparsely located across the study region without any clear spatial trends.

We plotted the mean proportions of cell area protected among the cells in different top fractions of Zonation solutions for each of the four main solutions (Fig. 7). For both of the European scale analyses the proportion protected did not seem to depend at all whether the cells were considered of high or low priority. Actually their pattern of distribution appeared near random. Instead, for the national scale analyses there was a consistent pattern of increasing protection with increasing rank in Zonation, for both the basic and phylogenetic diversity variants of Zonation. Topmost $1 \%$ fractions had almost twice as much area under protection as compared with the mean across the whole study region.

\section{Discussion and Conclusions}

We prioritized areas for conservation of hotspots of mammalian evolutionary history with a spatial prioritization tool. Majority of high priority areas for species conservation are also of high priority for the conservation of evolutionary history, but there are some regions where substantial differences occur between the two different goals. This implies that targeting species alone does not necessarily succeed in protection of hotspots of evolutionary history. Past research has found mixed evidence of such surrogacy relationships between protecting species and phylogenetic diversity (Polasky et al. 2001; Rodrigues and Gaston 2002; Sechrest et al. 2002; Forest et al. 2007; Spathelf and Waite 2007; Rodrigues et al. 2011). Our findings show that it makes a difference in what regions such comparisons are made: we found little difference between priorities around the Mediterranean, but much more 

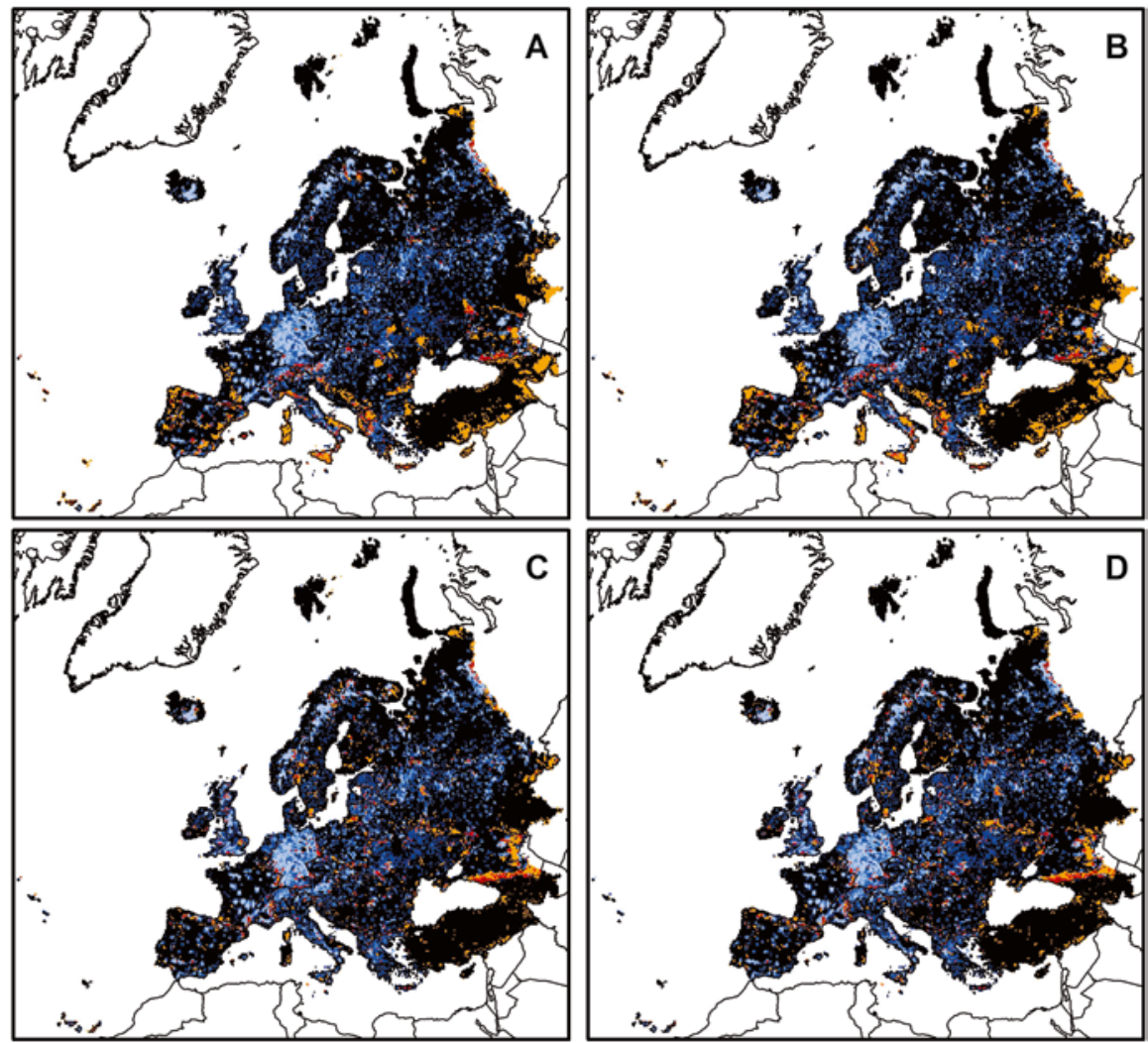

$\%$ protected area per cell

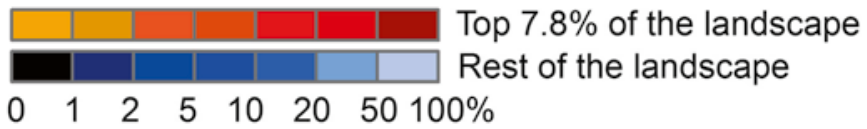

Fig. 6 Overlap of the area identified as priority for conservation with Zonation ( $7.8 \%$ of the land area in the study region) with currently protected areas (WDPA categories I-IV). The two different color scales indicate the proportion of each cell under protection: Light blue cells have more than half of their area protected, black cells have less than $1 \%$. The tones from yellow to dark red indicate the same thing, but for the cells belonging to the Zonation $7.8 \%$ top fractions. (a) Is the basic, core-area Zonation solution for our data where conservation value in Zonation optimization is only based on species richness normalised by range size. (b) Is the Zonation solution where the conservation value of a cell is weighted with the medium phylogenetic diversity, i.e. the inverse of the equivalent number of Rao's quadratic entropy for the local community in each cell is used as cell costs. (c) Shows the national level basic Zonation priorities and (d) is the national analysis with phylogenetic diversity included

e.g. in Scandinavia. Chazot et al. (chapter "Patterns of Species, Phylogenetic and Mimicry Diversity of Clearwing Butterflies in the Neotropics") found that phylogenetic diversity and species richness are less correlated in areas of low species richness, which might explain some of the patterns in our results as well. 
Basic Zonation

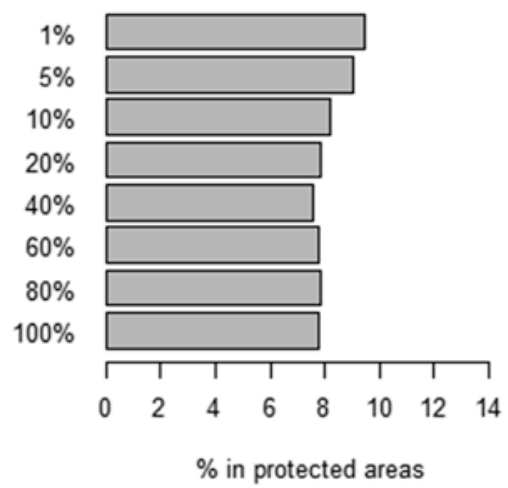

National basic

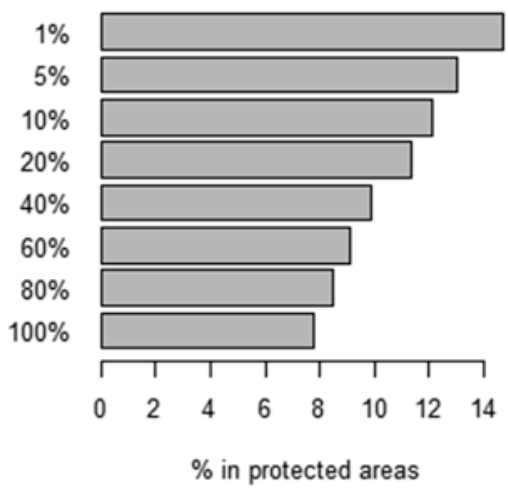

Phylogenetic diversity

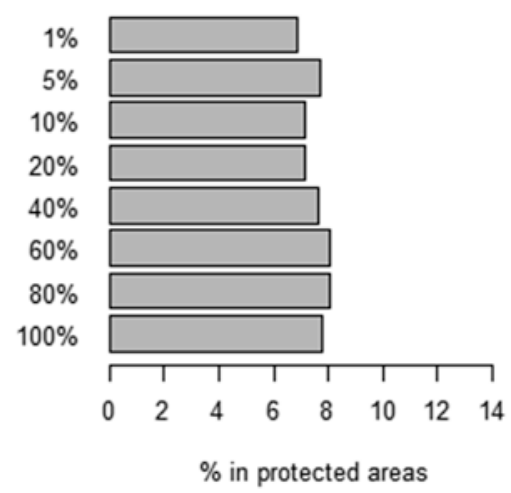

National phyl. diversity

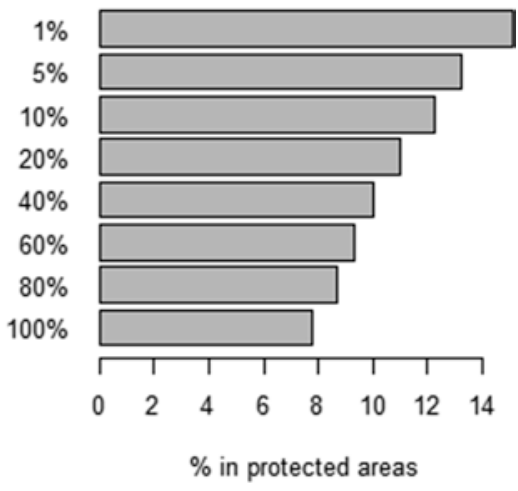

Fig. 7 The mean proportion of area protected in the different top fractions of the Zonation rankings. For example, out of the best $1 \%$ of the cells according to the continental scale phylogenetic diversity variant (with inverse of the equivalent number of Rao's QE as cell cost) approximately $7 \%$ of area is under protection, whereas in both of the National Zonation variants more than twice as much of the top priority area is under protection. The $100 \%$ bar indicates the overall mean of area protected across the whole study region, corresponding to $7.8 \%$ of land area

Mammals are a group of species with broad distributions even at the European scale. Such patterns cause the priorities to be strongly concentrated around southern parts of the study area, where the diversity gradients peak. Whenever such a region is subdivided into smaller administrative units, species ranges will typically extend over multiple units. And whenever distributional ranges cross boundaries, selecting areas complementary to each other within a subunit of a larger area is likely to lead to selecting areas as far as possible from each other: the Northern border will host mostly different species from those along the southern border. This so-called edge artefact (Moilanen et al. 2013) is important to consider when discussing the relevance of spatial scales in priority setting. 
A European scale prioritization is much more cost-efficient in covering species ranges, as compared with the national scale analyses that barely surpass a random selection (Fig. 4). National level prioritization is bound to be less cost-effective (Erasmus et al. 1999; Kark et al. 2009; Bladt et al. 2009) if all species, including the broadly distributed ones, must be conserved separately in each country. However, it would not be politically feasible to focus all conservation efforts to the Europeanscale hotspots either, because these cover disproportionate fractions of some countries, while leaving others virtually unprotected. Therefore, in reality a balanced compromise solution between the two extremes would be desirable, but such options are explored elsewhere (Moilanen and Arponen 2011; Moilanen et al. 2013).

Our results suggest that the amount of uncertainty related to the mammal phylogenies is not significant from the perspective of spatial prioritization of evolutionary hotspots. The differences between trees are minor and appear to occur in parts of the phylogeny with species that mainly occur in species rich communities, and thus the patterns of species distributions drive the prioritization and mask the impact of phylogenetic uncertainty. This is not to say that phylogenetic uncertainty in general would not matter in conservation prioritization. It may well be that for less well known taxa with higher uncertainty, or taxa with different phylogenetic structure and different kinds of patterns of spatial distributions of the species would show much higher variation in prioritization outcomes. The result could also be different for another conservation goal, e.g., if aiming at maximizing phylogenetic diversity across the study region (Rodrigues and Gaston 2002; Rodrigues et al. 2011) rather than considering it at the level of local community as we do here.

When including additional constraints to prioritization, such as a weighting based on phylogenetic diversity, some other aspect may have to be compromised and trade-offs sought, as priorities for different goals rarely perfectly coincide. In the case of the European mammals and alpha-level phylogenetic diversity, we found that the trade-offs were very reasonable, and indeed, negligible as compared with the losses incurred by restricting the prioritization to the national scale.

As expected, the mean phylogenetic diversity in cells prioritized by Zonation variants where phylogenetic diversity was included as a cost layer were higher than in those variants that did not include it. In relative terms the differences were not enormous (see Fig. 5), but one must consider that (1) Zonation can only work with the values that occur in the landscape, and in this case it had to select from a set of cells where the overall mean of phylogenetic diversity (measured as the equivalent number of Rao's quadratic entropy) was slightly larger than 5 and maximum was 7, and (2) the Core-area Zonation needs to retain core cells for all species, and cannot entirely give up on an "expensive" species - that is, a species occurring only in cells with very low phylogenetic diversity. Thus, the flexibility of the solutions strongly depends on the spatial patterns of species distributions and how they relate to the phylogenetic tree structure. For example, if species' range sizes are relatively small and overlap little, Zonation needs to retain a large number of cells to cover core distributions for all of them, and thus there is little flexibility in the solution even when variation in cell costs (or phylogenetic diversity) is high. If rare endemics happen to occur in cells with the highest phylogenetic diversity and all other species 
have very broad distributions, then that leaves quite a lot of flexibility for ranking the rest of the landscape.

We found the current network of protected areas to perform rather poorly with respect to representation of areas perceived as high priorities by the European scale Zonation solutions. The proportions of different fractions from Zonation solutions were covered by protected areas roughly equally, close to the overall mean percentage of protected area in the study region. In other words, current protected areas appear equivalent to a random allocation of sites when compared with the European scale Zonation priorities. However, as discussed earlier, prioritization at the European scale may not be a reasonable comparison, as conservation planning in the real world mainly happens at more local scales. The comparison to the national scale prioritization was more positive, with highest Zonation priorities almost twice as likely to be protected as the mean across the region. Even though less than $15 \%$ protection of the highest priorities is perhaps not an outcome to celebrate, it does indicate that at least according to some criteria, protected area allocation in Europe has not been fully opportunistic, and is not worse than random, as could be the case when the sites are biased towards areas of low economic interest (Ferrier 2002).

However, it is also important to remember, that even the best solution at national scale was only mildly better than a random selection (Fig. 4), making it another unreasonable baseline to compare against. It may well be, that the higher coincidence of protected areas with Zonation priorities is simply a consequence of countries preferably locating protected areas near borders, which coincides with spatial priorities due to the edge artefact mentioned above, rather than being a sign of costeffective protected area planning. Such a pattern was found in the Americas in a previous study (Moilanen et al. 2013). Analyses at higher data resolutions that include other taxa and different aspects of diversity are required to make more realistic and useful assessments of protected areas, but our first attempt does provide some interesting insight into these questions.

Conservation of evolutionary history is generally acknowledged to be important, although the debate on the alternative justifications for it is still ongoing (Rosauer and Mooers 2013; Winter et al. 2013). The underlying reason for its conservation will influence the practical goals and conservation priorities. Our analysis identifies priority regions for conserving high alpha-level phylogenetic diversity for mammals. Such an approach is typically justified on the basis of representing higher functional diversity (see section "Introduction"), but due to the correlation of QE with species richness it may also be closer to a species-based solution than some alternative ways of considering evolutionary history in conservation prioritization. Therefore, our results should not be taken as proof of an existing surrogacy relationship of species and phylogenetic diversity-based prioritizations, especially as also with our approach there were some regions with clear differences to species-based prioritization. An important notion regarding Zonation, or any prioritization tool, is that it does not inherently "know" what is desirable in conservation. It can only answer the questions it is posed, and it is up to the user that the questions make sense (see also Moilanen 2008). For example, merely adjusting the strength of the weighting (cost layer) in the current analysis will shift priorities to some extent. 
Similar prioritizations for different taxa are also quite likely to produce different outcomes. Conservation is always driven by value judgments (Vane-Wright and Coppock 2009), and there is even a risk of purposefully setting goals in a manner that produces desired spatial outcomes.

Since there necessarily are multiple potentially relevant objectives, a conservative, precautionary strategy would be to assess several of them and focus on areas where most priorities are in concordance, and consider as unimportant only the areas where no high priorities occur. However, in practice different types of conservation actions could be necessary to address the different objectives, and therefore the conflicts may be more apparent than real. For instance, regions with particularly low phylogenetic diversity may also be of conservation concern as they can represent areas of active diversification (Forest et al. 2007), but they might require different type of conservation from "museum" areas with relict species, as these areas and species in them might be threatened by very different processes.

Another open and closely related question is at what spatial scales should we operate when measuring and prioritizing evolutionary history? In our case the assumption was that phylogenetic diversity measured as the equivalent number of Rao's quadratic entropy of the local community was the relevant unit, but especially when assessing the diversity across the study region, the delineation of the study region will have an impact on priorities as described above, but also through "pruning" of the phylogenetic tree: A specific region will cover parts of a full phylogeny, and regional scale prioritization with such a partial tree may prioritize areas different from a global prioritization with a full tree.

Considering the amount of literature on conservation of evolutionary history in general, it is surprising how rarely it is considered in systematic conservation planning applications. Phylogenetic data are increasing and the modern computational prioritization tools are better able to account for such data even at broad scales and for large numbers of species. These developments facilitate the inclusion of phylogenetic diversity into conservation planning. We hope that it will become a routine part of spatial conservation prioritization procedures, and that the message will also better reach the broader public through active communication.

Acknowledgements A.A. was funded by the Academy of Finland (grant \#250126 to A.A.). LZ was funded by the ANR-BiodivERsA project CONNECT (ANR-11-EBID-002), as part of the ERA-Net BiodivERsA 2010 call. A part of the computations presented here were performed using the CIMENT infrastructure (https://ciment.ujf-grenoble.fr), which is supported by the RhôneAlpes region (GRANT CPER07_13 CIRA: http://www.ci-ra.org). We wish to thank L. Maiorano for the data compilation, W. Thuiller and T. Münckemüller for their programming support, as well as Mar Cabeza, 2 anonymous reviewers and Roseli Pellens for useful comments on the manuscript.

Open Access This chapter is distributed under the terms of the Creative Commons AttributionNoncommercial 2.5 License (http://creativecommons.org/licenses/by-nc/2.5/) which permits any noncommercial use, distribution, and reproduction in any medium, provided the original author(s) and source are credited.

The images or other third party material in this chapter are included in the work's Creative Commons license, unless indicated otherwise in the credit line; if such material is not included 
in the work's Creative Commons license and the respective action is not permitted by statutory regulation, users will need to obtain permission from the license holder to duplicate, adapt or reproduce the material.

\section{References}

Arponen A (2012) Prioritizing species for conservation planning. Biodivers Conserv 21:875-893. doi:10.1007/s10531-012-0242-1

Arponen A, Heikkinen RK, Thomas CD, Moilanen A (2005) The value of biodiversity in reserve selection: representation, species weighting, and benefit functions. Conserv Biol 19:20092014. doi:10.1111/j.1523-1739.2005.00218.x

Ball IR, Possingham HP, Watts M (2009) Marxan and relatives: software for spatial conservation prioritisation. In: Moilanen A, Wilson KH, Possingham HP (eds) Spatial conservation prioritisation: quantitative methods and computational tools. Oxford University Press, Oxford, pp 185-195

Bininda-Emonds ORP, Cardillo M, Jones KE et al (2007) The delayed rise of present-day mammals. Nature 446:507-512

Bladt J, Strange N, Abildtrup J et al (2009) Conservation efficiency of geopolitical coordination in the EU. J Nat Conserv 17:72-86. doi:10.1016/j.jnc.2008.12.003

Cadotte MW, Cardinale BJ, Oakley TH (2008) Evolutionary history and the effect of biodiversity on plant productivity. Proc Natl Acad Sci 105:17012-17017. doi:10.1073/pnas.0805962105

Cadotte M, Dinnage R, Tilman GD (2012) Phylogenetic diversity promotes ecosystem stability. Ecology 93:223-233. doi:10.1890/11-0426.1

Chao A, Chiu C-H, Jost L (2010) Phylogenetic diversity measures based on Hill numbers. Philos Trans R Soc Lond Ser B Biol Sci 365:3599-3609

Davies TJ, Buckley LB (2011) Phylogenetic diversity as a window into the evolutionary and biogeographic histories of present-day richness gradients for mammals. Philos Trans R Soc Lond Ser B Biol Sci 366:2414-2425

De Bello F, Lavergne S, Meynard CN et al (2010) The partitioning of diversity: showing Theseus a way out of the labyrinth. J Veg Sci 21:992-1000. doi:10.1111/j.1654-1103.2010.01195.x

Eklund J, Arponen A, Visconti P, Cabeza M (2011) Governance factors in the identification of global conservation priorities for mammals. Philos Trans R Soc Lond B Biol Sci 366:26612669. doi:10.1098/rstb.2011.0114

Erasmus BFN, Freitag S, Gaston KJ et al (1999) Scale and conservation planning in the real world. Proc R Soc Lond Ser B Biol Sci 266:315-319

Faith DP (1992) Conservation evaluation and phylogenetic diversity. Biol Conserv 61:1-10

Ferrier S (2002) Mapping spatial pattern in biodiversity for regional conservation planning: where to from here? Syst Biol 51:331-363. doi:10.1080/10635150252899806

Forest FF, Grenyer R, Rouget M et al (2007) Preserving the evolutionary potential of floras in biodiversity hotspots. Nature 445:757-760. doi:10.1038/nature05587

Fritz SA, Rahbek C (2012) Global patterns of amphibian phylogenetic diversity. J Biogeogr 39:1373-1382. doi:10.1111/j.1365-2699.2012.02757.x

Fritz SA, Bininda-Emonds ORP, Purvis A (2009) Geographical variation in predictors of mammalian extinction risk: big is bad, but only in the tropics. Ecol Lett 12:538-549

Isaac NJB, Turvey ST, Collen B et al (2007) Mammals on the EDGE: conservation priorities based on threat and phylogeny. PLoS One 2:e296. doi:10.1371/journal.pone.0000296

IUCN (2012) The IUCN Red List of threatened species. Version 2012.2. http://www.iucnredlist. org 
Jost L (2007) Partitioning diversity into independent alpha and beta components. Ecology 88:2427-2439

Kark S, Levin N, Grantham HS, Possingham HP (2009) Between-country collaboration and consideration of costs increase conservation planning efficiency in the Mediterranean Basin. Proc Natl Acad Sci U S A 106:15368-15373. doi:10.1073/pnas.0901001106

Kuhn TS, Mooers AØ, Thomas GH (2011) A simple polytomy resolver for dated phylogenies. Methods Ecol Evol 2:427-436. doi:10.1111/j.2041-210X.2011.00103.x

Leinster T, Cobbold CA (2012) Measuring diversity: the importance of species similarity. Ecology 93:477-489

Maiorano L, Amori G, Capula M et al (2013) Threats from climate change to terrestrial vertebrate hotspots in Europe. PLoS One 8:e74989. doi:10.1371/journal.pone.0074989

Margules CR, Pressey RL (2000) Systematic conservation planning. Nature 405:243-253. doi: $10.1038 / 35012251$

Margules C, Sarkar S (2007) Systematic conservation planning. Cambridge University Press, Cambridge, p 278

Moilanen A (2007) Landscape Zonation, benefit functions and target-based planning: unifying reserve selection strategies. Biol Conserv 134:571-579. doi:10.1016/j.biocon.2006.09.008

Moilanen A (2008) Two paths to a suboptimal solution - once more about optimality in reserve selection. Biol Conserv 141:1919-1923. doi:10.1016/j.biocon.2008.04.018

Moilanen A, Arponen A (2011) Administrative regions in conservation: balancing local priorities with regional to global preferences in spatial planning. Biol Conserv 144:1719-1725. doi:10.1016/j.biocon.2011.03.007

Moilanen A, Franco AMA, Early RI et al (2005) Prioritising multiple use landscapes for conservation: methods for large multi species planning problems. Proc R Soc Lond Ser B Biol Sci 272:1885-1891. doi:10.1098/rspb.2005.3164

Moilanen A, Kujala H, Leathwick JR (2009) The Zonation framework and software for conservation prioritization. In: Moilanen A, Wilson KH, Possingham HP (eds) Spatial conservation prioritisation: quantitative methods and computational tools. Oxford University Press, Oxford, pp 196-210

Moilanen A, Leppänen J, Meller L et al (2011) Spatial conservation planning framework and software Zonation v. 3.0: user manual. University of Helsinki, Helsinki, p 278

Moilanen A, Anderson BJ, Arponen A et al (2013) Edge artefacts and lost performance in national versus continental conservation priority areas. Divers Distrib 19:171-183. doi:10.1111/ddi.12000

Mouquet N, Devictor V, Meynard CN et al (2012) Ecophylogenetics: advances and perspectives. Biol Rev Camb Philos Soc 87:769-785. doi:10.1111/j.1469-185X.2012.00224.x

Pavoine S, Bonsall MB (2011) Measuring biodiversity to explain community assembly: a unified approach. Biol Rev Camb Philos Soc 2:792-812. doi:10.1111/j.1469-185X.2010.00171.x

Pavoine S, Ollier S, Dufour A-B, Crozier R (2005a) Is the originality of a species measurable? Ecol Lett 8:579-586. doi:10.1111/j.1461-0248.2005.00752.x

Pavoine S, Ollier S, Pontier D (2005b) Measuring diversity from dissimilarities with Rao's quadratic entropy: are any dissimilarities suitable? Theor Popul Biol 67:231-239

Polasky S, Csuti B, Vossler CA, Meyers SM (2001) A comparison of taxonomic distinctness versus richness as criteria for setting conservation priorities for North American birds. Biol Conserv 97:99-105

Pressey RL, Humphries CJ, Margules CR et al (1993) Beyond opportunism - key principles for systematic reserve selection. Trends Ecol Evol 8:124-128

Rao CR (1982) Diversity and dissimilarity coefficients: a unified approach. Theor Popul Biol 21:24-43

Rodrigues ASL, Gaston KLJ (2002) Maximising phylogenetic diversity in the selection of networks of conservation areas. Biol Conserv 105:103-111

Rodrigues ASL, Grenyer R, Baillie JEM et al (2011) Complete, accurate, mammalian phylogenies aid conservation planning, but not much. Philos Trans R Soc Lond B Biol Sci 366:2652-2660 
Rosauer DF, Mooers AO (2013) Nurturing the use of evolutionary diversity in nature conservation. Trends Ecol Evol 28:322-323. doi:10.1016/j.tree.2013.01.014

Schweiger O, Klotz S, Durka W, Kühn I (2008) A comparative test of phylogenetic diversity indices. Oecologia 157:485-495. doi:10.1007/s00442-008-1082-2

Sechrest W, Brooks TM, da Fonseca GAB et al (2002) Hotspots and the conservation of evolutionary history. Proc Natl Acad Sci U S A 99:2067-2071. doi:10.1073/pnas.251680798

Smith RJ, Veríssimo D, Isaac NJB, Jones KE (2012) Identifying Cinderella species: uncovering mammals with conservation flagship appeal. Conserv Lett 5:205-212. doi:10.1111/j.1755-263X.2012.00229.x

Spathelf M, Waite T a (2007) Will hotspots conserve extra primate and carnivore evolutionary history? Divers Distrib 13:746-751. doi:10.1111/j.1472-4642.2007.00386.x

UNEP I (2010) The World Database on Protected Areas (WDPA). UNEP-WCMC, Cambridge, www.protectedplanet.net

Vane-Wright RI, Coppock J (2009) Planetary awareness, worldviews and the conservation of biodiversity. In: Kellert SR, Speth JG (eds) The coming transformation. Values to sustain human and natural communities. Yale School of Forestry \& Environmental Studies, New Haven, pp 353-382

Vane-Wright RI, Humphries CJ, Williams PH (1991) What to protect? - systematics and the agony of choice. Biol Conserv 55:235-254

Winter M, Devictor V, Schweiger O (2013) Phylogenetic diversity and nature conservation: where are we? Trends Ecol Evol 28:199-204. doi:10.1016/j.tree.2012.10.015 